\title{
ON MINIMAL SINGULAR METRICS OF CERTAIN CLASS OF LINE BUNDLES WHOSE SECTION RING IS NOT FINITELY GENERATED
}

\author{
TAKAYUKI KOIKE
}

\begin{abstract}
Our interest is a regularity of a minimal singular metric of a line bundle. One main conclusion of our general result in this paper is the existence of smooth Hermitian metrics with semi-positive curvatures on the so-called Zariski's example of a line bundle defined over the blow-up of $\mathbb{P}^{2}$ at some twelve points. This is an example of a line bundle which is nef, big, not semi-ample, and whose section ring is not finitely generated. We generalize this result to the higher dimensional case when the stable base locus of a line bundle is a smooth hypersurface with a holomorphic tubular neighborhood.
\end{abstract}

\section{INTRODUCTION}

Our interest is a regularity of a minimal singular metric of a line bundle. One main conclusion of our general result in this paper is the existence of smooth Hermitian metrics with semi-positive curvatures on the so-called Zariski's example ([L, 2.3.A]).

Theorem 1.1 (Example 4.3). Let $C \subset \mathbb{P}^{2}$ be a smooth elliptic curve, $\pi: X \rightarrow \mathbb{P}^{2}$ the blowing-up at general twelve points $p_{1}, p_{2}, \ldots, p_{12} \in C, H$ the pulled back divisor of a line in $\mathbb{P}^{2}$, and let $D$ be the strict transform of $C$. Then the line bundle $L=\mathcal{O}_{X}(H+D)$ is semi-positive (i.e. L admits a smooth Hermitian metric with semi-positive curvature).

This $L$ is nef and big, however has a pathological property that $D \subset \mathrm{Bs}\left|L^{\otimes m}\right|$ holds for all $m \geq 1,\left|L^{\otimes m} \otimes \mathcal{O}_{X}(-D)\right|$ is globally generated for all $m \geq 1$, and that the section ring $\bigoplus_{m>0} H^{0}\left(X, L^{\otimes m}\right)$ of $L$ is not finitely generated. When the twelve points $p_{1}, p_{2}, \ldots, p_{12} \in C$ is special, the line bundle $L$ is semi-ample and thus it is semi-positive. Minimal singular metrics of a line bundle $L$ are metrics of $L$ with the mildest singularities among singular Hermitian metrics of $L$ whose local weights are plurisubharmonic. Minimal singular metrics have been introduced in [DPS00, 1.4] as a (weak) analytic analogue of the Zariski decomposition, and always exist when $L$ is pseudo-effective ([DPS00, 1.5]). The main theorem is as follows.

THEOREM 1.2. Let $X$ be a smooth projective variety, $D$ a smooth hypersurface of $X$, $L$ a pseudo-effective line bundle over $X$, and let $h_{\min }$ be a minimal singular metric of $L$. Assume that $L \otimes \mathcal{O}_{X}(-D)$ is semi-positive, $\left.\mathcal{O}_{X}(-D)\right|_{D}$ is ample, $\mathcal{O}_{D}\left(-K_{D}-\left.D\right|_{D}\right)$ is nef and big, and that $D$ has a holomorphic tubular neighborhood (i.e. an open neighborhood in $X$ which is biholomorphic to an open neighborhood of the zero section in the normal bundle $\left.N_{D / X}\right)$. Then $\left.h_{\min }\right|_{D} \not \equiv \infty$ holds if and only if $\left.L\right|_{D}$ is pseudo-effective, moreover in this case $\left.h_{\min }\right|_{D}$ is a minimal singular metric of $\left.L\right|_{D}$.

One of the typical cases of the situations in Theorem 1.2 is when $X$ is a surface and the self-intersection number $\left(D^{2}\right)$ is (sufficiently) negative. It is followed by a special

2010 Mathematics Subject Classification. 32J25; 14C20.

Key words and phrases. minimal singular metrics, tubular neighborhoods, Zariski's example. 
case of Grauert's theorem [G, Satz 7]: A smooth compact complex curve $D$ with genus $g$ embedded in a complex surface $X$ has a holomorphic tubular neighborhood if $\left(D^{2}\right)<$ $\min \{0,4-4 g\}$ holds. Thus, we can apply our main theorem to Zariski's example to obtain Theorem 1.1, and we also can show the existence of a smooth Hermitian metric with semi-positive curvature for the same type examples introduced by Mumford ([L, 2.3.A], or Example 4.3 here). When $\left.\left(L \otimes \mathcal{O}_{X}(-D)\right)\right|_{D}$ is ample, we can write down more concretely a minimal singular metric of $L$ around $D$ by using equilibrium metrics, which are special minimal singular metrics, of $\mathbb{R}$-line bundles $\left.\left(L \otimes \mathcal{O}_{X}(-t D)\right)\right|_{D}$ for $0 \leq t \leq 1$ (see Theorem 2.2 and Remark 3.4).

Another application of Theorem 1.2 we can expect is a concrete description of minimal singular metrics of a pseudo-effective line bundle which is not big. It is because, it follows from next corollary, which is a spacial case of Theorem 1.2, that we can apply Bergman kernel construction argument even when $L$ is not big but merely pseudo-effective. In more detail, the line bundle $\mathcal{O}_{\mathbb{P}(A \oplus L)}(1)$ in next Theorem 1.3 is big if we chose $A$ as an ample line bundle. Thus we can use Bergman kernel construction argument for this line bundle and we can study minimal singular metrics of $L$ itself by restricting argument.

THEOREM 1.3. Let $X$ be a smooth projective variety, A a semi-ample line bundle on $X$, and let $L$ be a pseudo-effective line bundle on $X$. Then the restriction of a minimal singular metric of $\mathcal{O}_{\mathbb{P}(A \oplus L)}(1)$ on $\mathbb{P}(A \oplus L)$ to the divisor $\mathbb{P}(L) \subset \mathbb{P}(A \oplus L)$ corresponding to the projection $A \oplus L \rightarrow L$ gives a minimal singular metric of $L$ on $X$ via the natural identification $\left(\mathbb{P}(L), \mathcal{O}_{\mathbb{P}(L)}(1)\right) \cong(X, L)$ (see Remark 2.3).

We can prove this corollary directly by constructing a minimal singular metric of $\mathcal{O}_{\mathbb{P}(A \oplus L)}(1)$ from fixed smooth Hermitian metrics of $A$ and $L$. In the proof of Theorem [1.2, we use the assumption on the existence of holomorphic tubular neighborhoods to reduce the situation in Theorem 1.2 to that in Theorem 1.3 . Since $L$ in Theorem 1.2 admits a singular Hermitian metric which is smooth on $X \backslash D$ and may be singular along $D$, all we have to do is to modify this metric around $D$. We will replace this metric on the tubular neighborhood of $D$ by the metric constructed in the situation of Theorem 1.3 .

The organization of the paper is as follows. In $\S 2$, we treat the case when $X$ has a suitable $\mathbb{P}^{1}$-bundle structure and $L$ is the relative hyperplane bundle, and we prove Theorem 1.3. In $\S 3$, we prove Theorem 1.2, Finally we give some examples in $\S 4$.

Acknowledgment. The author would like to thank his supervisor Prof. Shigeharu Takayama whose enormous support and insightful comments were invaluable during the course of his study. He also thanks Prof. Shin-ichi Matsumura who gave him invaluable comments. He is supported by the Grant-in-Aid for Scientific Research (KAKENHI No.25-2869) and the Grant-in-Aid for JSPS fellows. This work is supported by the Program for Leading Graduate Schools, MEXT, Japan.

\section{The $\mathbb{P}^{1}$-BUndLe CASE}

In this section, we treat the case when $X$ has a suitable $\mathbb{P}^{1}$-bundle structure and $L$ is the relative hyperplane bundle. Here we give a minimal singular metric of $L$ concretely by using equilibrium metrics of $\mathbb{R}$-line bundles of the base space of $X$. First we define the equilibrium metrics for smooth Hermitian metrics on pseudo-effective line bundles.

Definition 2.1. Let $X$ be a smooth projective variety, $L$ a pseudo-effective line bundle over $X$, and let $h=e^{-\varphi}$ be a smooth Hermitian metric on $L$. We denote by $h_{e}$ the 
equilibrium metric, whose local weight function $\varphi_{e}$ is defined by

$$
\varphi_{e}=\varphi+\sup ^{*}\{\psi: X \rightarrow \mathbb{R} \cup\{-\infty\} \mid \psi \text { is a } \varphi \text {-psh function, } \psi \leq 0\},
$$

where sup* stands for the upper semi-continuous regularization of the supremum.

Equilibrium metrics are minimal singular metrics ([DPS00, 1.5]). Using this notion, we prove the following theorem, a special case of Theorem 1.2 (see Remark 2.3 below).

THEOREM 2.2. Let $X$ be a smooth projective variety, $A$ an ample line bundle on $X$, and let $L$ be a pseudo-effective line bundle on $X$. Let $h_{L}=e^{-\varphi_{L}}$ be a smooth Hermitian metric of $L$ and let $h_{A}=e^{-\varphi_{A}}$ be a smooth Hermitian metric of $A$ satisfying $d d^{c} \varphi_{A}>0$. Fix a local coordinate system by $(z, x) \mapsto\left[z s_{A}^{*}(x)+s_{L}^{*}(x)\right] \in \mathbb{P}(A \oplus L)$, where $s_{A}^{*}$ and $s_{L}^{*}$ are local trivializations of $A^{-1}$ and $L^{-1}$, respectively. Then the metric of the relative hyperplane line bundle $\mathcal{O}_{\mathbb{P}(A \oplus L)}(1)$ on $\mathbb{P}(A \oplus L)$ defined by the local weights

$$
\tilde{\varphi}(z, x)=\log \max _{t \in[0,1]}|z|^{2 t} e^{\left(t \varphi_{A}+(1-t) \varphi_{L}\right)_{e}(x)}
$$

is a minimal singular metric, where $\left(t \varphi_{A}+(1-t) \varphi_{L}\right)_{e}$ is the local weight of the equilibrium metric associated to $h_{A}^{t} h_{L}^{1-t}$, which is a smooth Hermitian metric of the " $\mathbb{R}$-line bundle $A^{\otimes t} \otimes L^{\otimes(1-t)} "$.

REMARK 2.3. Here we use the notations of Theorem 2.2. Let us denote by $\tilde{X}$ the total space $\mathbb{P}(A \oplus L)$, by $\pi$ the projection $\tilde{X} \rightarrow X$, by $X^{\prime}$ the subset $\mathbb{P}(L)$ of $\tilde{X}$, and $X^{\prime \prime}$ the subset $\mathbb{P}(A)$. Then $\mathcal{O}_{\tilde{X}}\left(X^{\prime}\right)=\mathcal{O}_{\mathbb{P}(A \oplus L)}(1) \otimes \pi^{*} A^{-1}$ and $\left.\mathcal{O}_{\mathbb{P}(A \oplus L)}(1)\right|_{X^{\prime}}=\left.\pi^{*} L\right|_{X^{\prime}}$ hold as equalities of line bundles on $\tilde{X}$ and $X^{\prime}$, respectively. Therefore we can regard the restriction of a metric of $\mathcal{O}_{\mathbb{P}(A \oplus L)}(1)$ to $X^{\prime}$ as a metric of $L$, and by regarding $X^{\prime} \subset \tilde{X}$ as $\mathbb{P}\left(\mathcal{O}_{X}\right) \subset \mathbb{P}\left(\mathcal{O}_{X} \oplus\left(L^{-1} \otimes A\right)\right)$, we can identify $\tilde{X} \backslash X^{\prime \prime}$ and $X^{\prime}$ with the total space of the normal bundle $N_{X^{\prime} / \tilde{X}}$, which is isomorphic to the bundle $L \otimes A^{-1}$ via $\pi$, and its zero-section.

From now on, we prove Theorem 2.2. We denote by $\tilde{X}$ the variety $\mathbb{P}(A \oplus L)$, by $\pi: \tilde{X} \rightarrow X$ the canonical projection mapping, and by $\tilde{L}$ the relative plane line bundle $\mathcal{O}_{\mathbb{P}(A \oplus L)}(1)$ on $\tilde{X}$. Here we denote by $U$ the domain of definition of $s_{A}^{*}, s_{L}^{*}$ and $x$. We also use the smooth Hermitian metric $\tilde{h}_{\infty}=e^{-\tilde{\varphi}_{\infty}}$ of $\tilde{L}$, whose local weight is defined as $\tilde{\varphi}_{\infty}(z, x)=\log \left(|z|^{2} e^{\varphi_{A}(x)}+e^{\varphi_{L}(x)}\right)$. To prove Theorem 2.2, it is sufficient to show the following two propositions.

Proposition 2.4 (Plurisubharmonicity of $\tilde{\varphi}$ ). The function

$$
\tilde{\varphi}(z, x)=\log \max _{t \in[0,1]}|z|^{2 t} e^{\left(t \varphi_{a}+(1-t) \varphi_{L}\right)_{e}(x)}
$$

is plurisubharmonic and $\left\{e^{-\tilde{\varphi}}\right\}$ glue up to define a singular Hermitian metric of $\tilde{L}$.

Proposition 2.5 (Minimal singularity of $\tilde{\varphi}$ ). There is a constant $C$ such that $\left(\tilde{\varphi}_{\infty}\right)_{e} \leq$ $\tilde{\varphi}+C$ holds.

2.1. Proof of Proposition 2.4. Since $\log |z|^{2 t} e^{\left(t \varphi_{a}+(1-t) \varphi_{L}\right)_{e}(x)}$ is plurisubharmonic and a local weight of a singular Hermitian metric of $\tilde{L}$ for each $t \in[0,1]$, it is sufficient to show that $\tilde{\varphi}$ is upper semi-continuous, and it follows immediately from following Lemma 2.7 . 
Let us denote by $\psi_{t}$ the function

$$
\sup ^{*}\left\{\psi: X \rightarrow \mathbb{R} \cup\{-\infty\} \mid \psi \text { is a }\left(t \varphi_{A}+(1-t) \varphi_{L}\right) \text {-psh function, } \psi \leq 0\right\} .
$$

For proving Lemma 2.7, we need the following lemma.

LEMMA 2.6.

(1) The sequence $\left\{\frac{\psi_{t}}{1-t}\right\}_{t \in[0,1]}$ is monotonically increasing with respect to $t$.

(2) For all $t \in[0,1), \lim _{s \downarrow t} \frac{\psi_{s}}{1-s}=\frac{\psi_{t}}{1-t}$ holds.

Proof. (1) Let $t \leq s$ be elements of $[0,1]$. Since

$$
s \varphi_{A}+(1-s) \varphi_{L}+\frac{1-s}{1-t} \psi_{t}=\frac{1-s}{1-t}\left(t \varphi_{A}+(1-t) \varphi_{L}\right)_{e}+\frac{s-t}{1-t} \varphi_{A}
$$

holds, $\frac{1-s}{1-t} \psi_{t}$ is a $\left(s \varphi_{A}+(1-s) \varphi_{L}\right)$-psh function. As $\frac{1-s}{1-t} \psi_{t} \leq 0, \frac{1-s}{1-t} \psi_{t} \leq \psi_{s}$ holds.

(2) According to (1), it is sufficient to show that $\lim _{s \downarrow t} \frac{\psi_{s}}{1-s} \leq \frac{\psi_{t}}{1-t}$ holds. Since the sequence $\frac{s}{1-s} \varphi_{A}+\varphi_{L}+\frac{\psi_{s}}{1-s}\left(=\frac{1}{1-s}\left(s \varphi_{A}+(1-s) \varphi_{L}\right)_{e}\right)$ is monotonically increasing psh functions, the limit $\frac{t}{1-t} \varphi_{A}+\varphi_{L}+\lim _{s \downarrow t} \frac{\psi_{s}}{1-s}$ is also psh. As $\lim _{s \downarrow t} \frac{\psi_{s}}{1-s} \leq 0,(1-t) \lim _{s \downarrow t} \frac{\psi_{s}}{1-s} \leq$ $\psi_{t}$ holds.

Lemma 2.7. The function $F: \mathbb{C} \times U \times[0,1] \rightarrow \mathbb{R} \cup\{-\infty\}$ defined by $F(z, x, t)=$ $\left(t \varphi_{A}+(1-t) \varphi_{L}\right)_{e}(x)+t \log |z|^{2}$ is upper semi-continuous.

Proof. Let us set the function $H: U \times[0,1] \rightarrow \mathbb{R} \cup\{-\infty\}$ as $H(x, t)=\frac{\psi_{t}(x)}{1-t}$. Since $F(z, x, t)$ is a sum of upper semi-continuous functions and $(1-t) H(x, t)$, it is sufficient to show that $H$ is upper semi-continuous. Let us fix an element $\left(x_{0}, t_{0}\right) \in U \times[0,1)$ and sufficiently small positive number $\varepsilon$. Then, by Lemma 2.6 (1),

$$
\limsup _{(x, t) \mapsto\left(x_{0}, t_{0}\right)} H(x, t)=\lim _{r \downarrow 0} \sup _{\substack{x-x_{0}|<r\\| t-t_{0} \mid<r}} H(x, t) \leq \lim _{r \downarrow 0} \sup _{\left|x-x_{0}\right|<r} H\left(x, t_{0}+\varepsilon\right)
$$

holds. As $H\left(-, t_{0}+\varepsilon\right)=\frac{\psi_{t_{0}+\varepsilon}}{1-\left(t_{0}+\varepsilon\right)}$ is upper semi-continuous, we obtain an inequality $\lim \sup _{(x, t) \mapsto\left(x_{0}, t_{0}\right)} H(x, t) \leq H\left(x_{0}, t_{0}+\varepsilon\right)$. By Lemma 2.6 (2), we can show that the equality $\lim \sup _{(x, t) \mapsto\left(x_{0}, t_{0}\right)} H(x, t) \leq H\left(x_{0}, t_{0}\right)$ holds. We can also show this inequality when $t_{0}=1$ by the same argument, and this shows the lemma.

2.2. Proof of Proposition 2.5. Next we prove Proposition 2.5. Let us fix a (sufficiently positive) Kähler metric $\omega$ of $X$ and define

$$
\tilde{\omega}=\pi^{*} \omega+d d^{c} \log \left(|z|^{2} e^{\varphi_{A}}+e^{\varphi_{L}}\right)-\frac{|z|^{2} e^{\varphi_{A}} \pi^{*} d d^{c} \varphi_{A}+e^{\varphi_{L}} \pi^{*} d d^{c} \varphi_{L}}{|z|^{2} e^{\varphi_{A}}+e^{\varphi_{L}}},
$$

where $d d^{c}=\frac{\sqrt{-1}}{2 \pi} \partial \bar{\partial}$. This $\tilde{\omega}$ defines a global smooth $(1,1)$-form on $\tilde{X}$, since $d d^{c} \log \left(|z|^{2} e^{\varphi_{A}}+\right.$ $\left.e^{\varphi_{L}}\right)$ is the curvature form of the smooth Hermitian metric of $\tilde{L}$ associated to the Finsler metric of $A \oplus L$ induced from $h_{A}$ and $h_{L}$, and both the coefficients $|z|^{2} e^{\varphi_{A}} /\left(|z|^{2} e^{\varphi_{A}}+e^{\varphi_{L}}\right)$ of $\pi^{*} d d^{c} \varphi_{A}$ and $e^{\varphi_{L}} /\left(|z|^{2} e^{\varphi_{A}}+e^{\varphi_{L}}\right)$ of $\pi^{*} d d^{c} \varphi_{L}$ glue up to define $\mathbb{R}$-valued functions defined on whole $\tilde{X}$.

LEMMA 2.8. The form $\tilde{\omega}$ and the measures $d V_{\omega}=\frac{\omega^{n}}{n !}$ of $X$ and $d V_{\tilde{\omega}}=\frac{\tilde{\omega}^{n+1}}{(n+1) !}$ of $\tilde{X}$ satisfy the following properties when $\omega$ is sufficiently positive.

(1) $\tilde{\omega}$ is a smooth strictly positive $(1,1)$-form on $\tilde{X}$. 
(2) For all $x \in X,\left.\int_{z \in \pi^{-1}(x)} d V_{\tilde{\omega}}\right|_{\pi^{-1}(x)}=1$ holds.

(3) For all $\mathbb{R}$-valued measurable function $F$ on $\tilde{X}$, the equation

$$
\int_{(z, x) \in \tilde{X}} F(z, x) d V_{\tilde{\omega}}=\int_{x \in X}\left(\left.\int_{z \in \pi^{-1}(x)} F(z, x) d V_{\tilde{\omega}}\right|_{\pi^{-1}(x)}\right) d V_{\omega}
$$

holds.

(4) Moreover, when $F$ depends only on $x$ and $|z|$, an equation

$$
\int_{(z, x) \in \tilde{X}} F(z, x) d V_{\tilde{\omega}}=\int_{x \in X}\left(\int_{0}^{\infty} \frac{2 r G(r, x) e^{\varphi_{A}(x)+\varphi_{L}(x)}}{\left(r^{2} e^{\varphi_{A}(x)}+e^{\varphi_{L}(x)}\right)^{2}} d r\right) d V_{\omega}
$$

holds, where $G$ is the function such that $G(|z|, x)=F(z, x)$ holds.

PROOF. By straight forward computations, we can obtain the formula

$$
\tilde{\omega}=\pi^{*} \omega+C(\eta \wedge \bar{\eta}+d z \wedge \bar{\eta}+\eta \wedge d \bar{z}+d z \wedge d \bar{z}),
$$

where $C=\frac{\sqrt{-1}}{2 \pi} \frac{e^{\varphi_{A}+\varphi_{L}}}{\left(|z|^{2} e^{\varphi} A+e^{\varphi} L\right)^{2}}$ and $\eta=z \partial\left(\varphi_{A}-\varphi_{L}\right)$. From this formula, it is shown that $\tilde{\omega}^{n+1}=(n+1) C d z \wedge d \bar{z} \wedge\left(\pi^{*} \omega\right)^{n}$ holds, which shows the lemma.

We also use the following lemma, which can be proved by straight forward computations.

LEMMA 2.9.

$$
-\log \int_{z \in \pi^{-1}(x)}|z|^{2 t} e^{-\tilde{\varphi}_{\infty}(z, x)} d V_{\left.\tilde{\omega}\right|_{\pi^{-1}(x)}}=t \varphi_{A}(x)+(1-t) \varphi_{L}(x)-\log \frac{\Gamma(1+t) \Gamma(2-t)}{4}
$$

holds for all $t \in[0,1]$ and $x \in X$, where $\Gamma$ stands for the Gamma function.

The following lemma can be shown by using the approximation theorem [D2, 13.21].

LEMmA 2.10. Let $Y$ be a smooth projective variety, $d V_{Y}$ a smooth volume form of $Y, M$ a pseudo-effective line bundle over $Y$, and let $h_{M}=e^{-\psi_{\infty}}$ be a smooth Hermitian metric of $M$. Fix points $y_{0}, y_{1}, \ldots y_{N} \in Y$ and local coordinates systems around each $y_{j}$ such that $\bigcup_{j=0}^{N}\left\{y|| y-y_{j} \mid<\frac{1}{\sqrt{\pi}}\right\}=Y$ holds. Let $h_{M, 1}=e^{-\psi_{1}}$ and $h_{M, 2}=e^{-\psi_{2}}$ be singular Hermitian metrics given by

$$
\begin{aligned}
& \psi_{1}=\psi_{\infty}+\sup ^{*}\left\{\left.\frac{1}{m} \log |f|_{h_{M}^{m}}^{2}\left|m \in \mathbb{N}, f \in H^{0}(Y, m M), \log \right| f\right|_{h_{M}^{m}} ^{2} \leq 0\right\}, \\
& \psi_{2}=\psi_{\infty}+\sup ^{*}\left\{\left.\frac{1}{m} \log |f|_{h_{M}^{m}}^{2}\left|m \in \mathbb{N}, f \in H^{0}(Y, m M), \int_{Y}\right| f\right|_{h_{M}^{m}} ^{2} d V_{Y} \leq 1\right\} .
\end{aligned}
$$

Let $C^{\prime}=C_{1}^{\prime}+C_{2}^{\prime}$ with $C_{1}^{\prime}=\max _{j}\left(\max _{\left|y-y_{j}\right| \leq \frac{2}{\sqrt{\pi}}} \psi_{\infty}(y)-\min _{\left|y-y_{j}\right| \leq \frac{2}{\sqrt{\pi}}} \psi_{\infty}(y)\right)$ and $C_{2}^{\prime}=$ $\log \max _{j} \max _{\left|y-y_{j}\right| \leq \frac{2}{\sqrt{\pi}}} \frac{d \lambda}{d V_{Y}}$, where $d \lambda$ is the Euclidean measure. Then an inequality $\psi_{2}-$ $C^{\prime} \leq \psi_{1} \leq\left(\psi_{\infty}\right)_{e}$ holds. Moreover, if $M$ is big, then an inequality $\psi_{2}-C^{\prime} \leq \psi_{1} \leq\left(\psi_{\infty}\right)_{e} \leq$ $\psi_{2}$ holds.

Proof of Proposition 2.5. (1) We fix points $x_{0}, x_{1}, \ldots x_{N} \in X$ and local coordinates systems around each $x_{j}$ such that $\bigcup_{j=0}^{N}\left\{x|| x-x_{j} \mid<\frac{1}{\sqrt{\pi}}\right\}=X$ holds. Let us denote by 
$\varphi_{\infty, t}$ the weight of the "smooth Hermitian metric" $t \varphi_{A}+(1-t) \varphi_{L}-\log \frac{\Gamma(1+t) \Gamma(2-t)}{4}$ of $t A+(1-t) L$. We let $C=C_{1}+C_{2}+\log 2$ with

$$
\begin{aligned}
& C_{1}=\max _{j}\left(\max _{\substack{\left|x-x_{j}\right| \leq \frac{2}{\sqrt{\pi}} \\
t \in[0,1]}} \tilde{\varphi}_{\infty, t}(x)-\min _{\substack{\left|x-x_{j}\right| \leq \frac{2}{\sqrt{\pi}} \\
t \in[0,1]}} \tilde{\varphi}_{\infty, t}(x)\right), \\
& C_{2}=\log \max _{j} \max _{\left|x-x_{j}\right| \leq \frac{2}{\sqrt{\pi}}} \frac{d \lambda}{d V_{\omega}} .
\end{aligned}
$$

Since $\left(\varphi_{\infty, t}\right)_{e}=\left(t \varphi_{A}+(1-t) \varphi_{L}\right)_{e}-\log \frac{\Gamma(1+t) \Gamma(2-t)}{4}$ holds, it is sufficient to show that

$$
\left(\tilde{\varphi}_{\infty}\right)_{e} \leq \log \max _{t \in[0,1]}|z|^{2 t} e^{\left(\varphi_{\infty, t}\right)_{e}(x)}+C
$$

holds. According to the last part of Lemma 2.10, this is reduced to show that for each $F \in H^{0}(\tilde{X}, m \tilde{L})$ such that $\int_{\tilde{X}}|F|^{2} e^{-m \tilde{\varphi}_{\infty}} d V_{\tilde{\omega}} \leq 1$, an inequality

$$
\frac{1}{m} \log |F|^{2} \leq \log \max _{t \in[0,1]}|z|^{2 t} e^{\left(\varphi_{\infty, t}\right)_{e}(x)}+C
$$

holds.

(2) We show the last inequality. The holomorphic section $F(z, x)$ can be expanded as $F(z, x)=\sum_{\ell=0}^{m} z^{\ell} f_{\ell}(x)$ with $f_{\ell} \in H^{0}(X, \ell A+(m-\ell) L)$. We first show that an inequality

$$
\int_{\tilde{X}}\left|z^{\ell} f_{\ell}\right|^{2} e^{-m \tilde{\varphi}_{\infty}} d V_{\tilde{\omega}} \leq 1
$$

holds for $\ell=1,2, \ldots, m$. For proving $(*)$, we use an inequality

$$
\left|f_{\ell}(x)\right|^{2}=\left|\frac{1}{\ell !} \frac{\partial^{\ell}}{\partial z^{\ell}} F(0, x)\right|^{2} \leq \frac{1}{2 \pi} \int_{0}^{2 \pi} \frac{\left|F\left(r e^{\sqrt{-1} \theta}, x\right)\right|^{2}}{r^{2 \ell}} d \theta
$$

for each positive number $r$. We denote by $\tilde{\varphi}_{\infty}(r, x)$ the function such that $\tilde{\varphi}_{\infty}(|z|, x)=$ $\tilde{\varphi}_{\infty}(z, x)$ holds. By multiplying the metric terms and integrating these with $r$, we obtain the following inequality.

$$
\begin{aligned}
& \int_{0}^{\infty} \frac{2 r\left(r^{2 \ell}\left|f_{\ell}(x)\right|^{2} e^{-m \tilde{\varphi}_{\infty}(r, x)}\right) e^{\varphi_{A}(x)+\varphi_{L}(x)}}{\left(r^{2} e^{\varphi_{A}(x)}+e^{\varphi_{L}(x)}\right)^{2}} d r \\
\leq & \frac{1}{2 \pi} \int_{0}^{\infty}\left(\int_{0}^{2 \pi} \frac{2 r\left(\left|F\left(r e^{\sqrt{-1} \theta}, x\right)\right|^{2} e^{-m \tilde{\varphi}_{\infty}(r, x)}\right) e^{\varphi_{A}(x)+\varphi_{L}(x)}}{\left(r^{2} e^{\varphi_{A}(x)}+e^{\varphi_{L}(x)}\right)^{2}} d \theta\right) d r \\
= & \left.\int_{z \in \pi^{-1}(x)}|F(z, x)|^{2} e^{-m \tilde{\varphi}(z, x)} d V_{\tilde{\omega}}\right|_{\pi^{-1}(x)} .
\end{aligned}
$$

This inequality and Lemma $2.8(2),(3),(4)$ implies the inequality (*). 
Then, by Lemma 2.8 (3),

$$
\begin{aligned}
1 & \geq \int_{(z, x) \in \tilde{X}}\left|z^{\ell} f_{\ell}(x)\right|^{2} e^{-m \tilde{\varphi}_{\infty}(z, x)} d V_{\tilde{\omega}} \\
& =\int_{x \in X}\left|f_{\ell}(x)\right|^{2}\left(\left.\int_{z \in \pi^{-1}(x)}\left|z^{\ell}\right|^{2} e^{-m \tilde{\varphi}_{\infty}(z, x)} d V_{\tilde{\omega}}\right|_{\pi^{-1}(x)}\right) d V_{\omega} \\
& =\int_{x \in X}\left|f_{\ell}(x)\right|^{2}\left(\left(\left.\int_{z \in \pi^{-1}(x)}\left(|z|^{2 \frac{\ell}{m}} e^{-\tilde{\varphi}_{\infty}(z, x)}\right)^{m} d V_{\tilde{\omega}}\right|_{\pi^{-1}(x)}\right)^{\frac{1}{m}} \cdot\left(\left.\int_{z \in \pi^{-1}(x)} 1^{\frac{m}{m-1}} d V_{\tilde{\omega}}\right|_{\pi^{-1}(x)}\right)^{\frac{m-1}{m}}\right)^{m} d V_{\omega} \\
& \geq \int_{x \in X}\left|f_{\ell}(x)\right|^{2}\left(\int_{z \in \pi^{-1}(x)}|z|^{2 \frac{\ell}{m}} e^{-\tilde{\varphi}_{\infty}(z, x)}\right)^{m} d V_{\omega}
\end{aligned}
$$

holds. Therefore, by Lemma 2.9. $\int_{x \in X}\left|f_{\ell}(x)\right|^{2} e^{-m \varphi_{\infty, t}(x)} d V_{\omega} \leq 1$ holds for $t=\frac{\ell}{m}$. Then by Lemma 2.10, we obtain an inequality $\frac{1}{m} \log \left|f_{\ell}\right|^{2} \leq\left(\varphi_{\infty, t}\right)_{e}+C_{1}+C_{2}$. Thus

$$
\begin{aligned}
& \frac{1}{m} \log |F(z, x)|^{2} \\
\leq & \frac{1}{m} \log \sum_{l=0}^{m}\left|z^{\ell} f_{\ell}(x)\right|^{2} \\
\leq & \frac{1}{m} \log \left((m+1) \max _{\ell}\left|z^{\ell} f_{\ell}(x)\right|^{2}\right) \\
= & \frac{1}{m} \log (m+1)+\log _{0 \leq \ell \leq m} \max _{0 \leq\left.\right|^{2} \frac{\ell}{m}}\left|f_{\ell}(x)\right|^{\frac{2}{m}} \\
\leq & \log 2+\log \max _{t \in[0,1]}|z|^{2 t} e^{\left(\varphi_{\infty, t}\right)_{e}(x)+C_{1}+C_{2}} \\
= & \log \max _{t \in[0,1]}|z|^{2 t} e^{\left(\varphi_{\infty, t}\right)_{e}(x)}+C
\end{aligned}
$$

holds.

\section{Proof of Theorem 1.2}

In this section, we prove Theorem 1.2, We first prove Theorem 1.3, a special case of Theorem 1.2.

Proof of Theorem 1.3. In order to show Theorem 1.3, it is sufficient to consider the singular Hermitian metric with local weight function $\log \left(|z|^{2} e^{\varphi_{A}(x)}+e^{\varphi_{L}(x)}\right)$ where $(z, x)$ is the coordinates just as in Theorem 2.2 and $\varphi_{A}, \varphi_{L}$ is the local weight of a minimal singular metric of $A, L$, respectively. Though this metric does not have minimal singularity in general, it is an extension of the minimal singular metric of $\left.\mathcal{O}_{\mathbb{P}(A \oplus L)}(1)\right|_{\mathbb{P}(L)}$.

Proof of Theorem 1.2. Let $X$ be a smooth projective variety, $D$ a 1-codimensional smooth subvariety of $X$, and let $L$ be a pseudo-effective line bundle over $X$. We assume that $A=L \otimes \mathcal{O}_{X}(-D)$ is semi-positive and that there is an open neighborhood $U$ of $D \subset X$ biholomorphic to an open neighborhood $U^{\prime}$ of the zero section of the normal bundle $N_{D / X}$. 
Here we may assume that $U^{\prime}=\left\{\left.\xi \in N_{D / X}|| \xi\right|_{h_{X / D}}<\varepsilon_{0}\right\}$ for some smooth Hermitian metric $h_{X / D}$ with negative curvature of $N_{D / X}$ and a positive number $\varepsilon_{0}$.

Since $\left.L\right|_{D}$ has no singular Hermitian metric of with psh local weights (which is not identically equal to $-\infty$ ) when $\left.L\right|_{D}$ is not pseudo-effective, all we have to do is showing the existence of a singular Hermitian metric of $L$ with psh local weights which is an extension of a minimal singular metric of $\left.L\right|_{D}$ assuming $\left.L\right|_{D}$ is pseudo-effective but not big. We set $X^{\prime}$ as the total space $\pi: \mathbb{P}\left(\left.\left.L\right|_{D} \oplus A\right|_{D}\right) \rightarrow D$ and $L^{\prime}$ as the relative hyperplane bundle $\mathcal{O}_{\mathbb{P}\left(\left.\left.L\right|_{D} \oplus A\right|_{D}\right)}(1)$. Let us fix a minimal singular metric $h_{L^{\prime}}=e^{-\varphi_{L^{\prime}}}$ of $L^{\prime}$. We set $V^{\prime}$ as the subset $\left\{\left.\xi \in N_{D / X}|| \xi\right|_{h_{X / D}}<\frac{\varepsilon_{0}}{2}\right\}$. By Remark 2.3, we can regard $U^{\prime}$ and $V^{\prime}$ be neighborhoods of $D^{\prime}=\mathbb{P}\left(\left.L\right|_{D}\right) \subset X^{\prime}$. We denote by $V$ the set $f\left(V^{\prime}\right) \subset U$, where $f: U^{\prime} \rightarrow U$ is the biholomorphic mapping.

By Proposition 3.1 below, there exists a line bundle $F$ on $U^{\prime}$ which admits a flat structure and $\left.f^{*}\left(\left.L\right|_{U}\right) \cong L^{\prime}\right|_{U^{\prime}} \otimes F$ holds. We fix a flat metric $h_{F}=e^{-\varphi_{F}}$ of $F$. By choosing appropriate local trivialization, we may assume $\varphi_{F} \equiv 0$. Thus we can regard $\left(f^{-1}\right)^{*} \varphi_{L^{\prime}}$ as the local weight function of the singular Hermitian metric $\left(f^{-1}\right)^{*} h_{L^{\prime}} h_{F}$ of $\left.L\right|_{U}$. To show the theorem, according to Theorem 1.3, it is sufficient to construct a singular Hermitian metric $e^{-\varphi_{L}}$ of $L$ with $d d^{c} \varphi_{L} \geq 0$ and $\left.\varphi_{L}\right|_{V}=\left.\left(f^{-1}\right)^{*} \varphi_{L^{\prime}}\right|_{V^{\prime}}$ holds. Let $h_{A}=$ $e^{-\varphi_{A}}$ be a smooth Hermitian metric of $A$ with $d d^{c} \varphi_{A} \geq 0$ and let $f_{D} \in H^{0}\left(X, \mathcal{O}_{X}(D)\right)$ be a section which vanishes only on $D$. Without loss of generality, we may assume $\varphi_{A} \geq 0,\left(f^{-1}\right)^{*} \varphi_{L^{\prime}} \leq-1$ holds on each fixed open set $W_{j}(j=1,2, \ldots, N)$ covering the whole $U$, and $\log \left|f_{D}\right|^{2} \geq-1$ holds on each intersection $W_{j} \cap(\bar{U} \backslash V)$. We define $\varphi_{L}$ as the function $\max \left\{\varphi_{A}+\log \left|f_{D}\right|^{2},\left(f^{-1}\right)^{*} \varphi_{L^{\prime}}\right\}$ on each $W_{j} \cap U$. Since $\varphi_{L}=\varphi_{A}+\log \left|f_{D}\right|^{2}$ holds on each intersection $W_{j} \cap(\bar{U} \backslash V), e^{-\varphi_{L}}$ on $U$ and $e^{-\left(\varphi_{A}+\log \left|f_{D}\right|^{2}\right)}$ on $X \backslash V$ glue up to define a new singular Hermitian metric of $L$, which proves the theorem.

Proposition 3.1. There is a line bundle $E$ on $D^{\prime}$ such that $c_{1}(E)=0$ and $f^{*}\left(\left.L\right|_{U} \cong \cong\right.$ $\left.\left(L^{\prime} \otimes \pi^{*} E\right)\right|_{U^{\prime}}$ hold.

In order to prove this proposition, we use the following form of Rossi's theorem [R, Theorem 3].

LEMma 3.2 (a version of Rossi's theorem). The natural map $H^{1}\left(U^{\prime}, \mathcal{O}_{U^{\prime}}\right) \rightarrow H^{1}\left(U^{\prime}, \mathcal{O}_{U^{\prime}} / I_{D^{\prime}}^{n}\right)$ is injective for some $n \geq 1$, where $I_{D^{\prime}}$ the defining ideal sheaf of $D \subset U$.

We first use Lemma 3.2 and show Proposition 3.1.

Proof of Proposition 3.1. The projection $\pi: U^{\prime} \rightarrow D$ and the injection $i: D^{\prime} \rightarrow U^{\prime}$ induce the maps $\pi^{*}: H^{1}\left(D^{\prime}, \mathcal{O}_{D^{\prime}}\right) \rightarrow H^{1}\left(U^{\prime}, \mathcal{O}_{U^{\prime}}\right)$ and $i^{*}: H^{1}\left(U^{\prime}, \mathcal{O}_{U^{\prime}}\right) \rightarrow H^{1}\left(D^{\prime}, \mathcal{O}_{D^{\prime}}\right)$, respectively. Since $\pi \circ i=\mathrm{id}_{D^{\prime}}, \pi^{*}$ is injective.

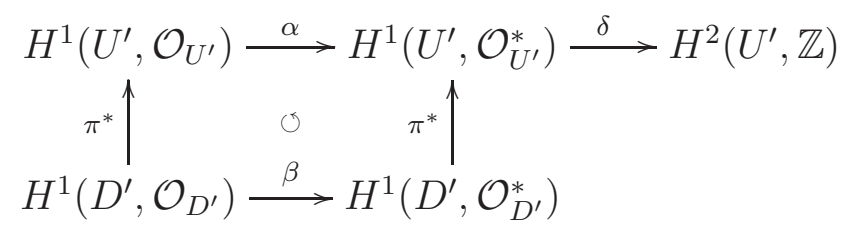

We first check that $\left.f^{*}\left(\left.L\right|_{U}\right) \otimes L^{\prime}\right|_{U^{\prime}} ^{-1}$ is topologically trivial line bundle. Indeed, $\left(f^{*}\left(\left.L\right|_{U}\right) \otimes\right.$ $\left.L^{\prime} \mid \begin{array}{l}-1 \\ U^{\prime}\end{array}\right)\left.\right|_{D^{\prime}}$ is the trivial bundle and $i \circ \pi$ is homotopic to $\operatorname{id}_{U^{\prime}}$. Thus we conclude that $\delta\left(\left.f^{*}\left(\left.L\right|_{U}\right) \otimes L^{\prime}\right|_{U^{\prime}} ^{-1}\right)=0$ and we can take an element $\xi \in H^{1}\left(U^{\prime}, \mathcal{O}_{U^{\prime}}\right)$ satisfying $\alpha(\xi)=$ $\left.f^{*}\left(\left.L\right|_{U}\right) \otimes L^{\prime}\right|_{U^{\prime}} ^{-1}$. When $\xi$ lies in the image of $\pi^{*}$, we can take an element $\eta \in H^{1}\left(D^{\prime}, \mathcal{O}_{D^{\prime}}\right)$ 
such that $\pi^{*}(\eta)=\xi$ holds. In this case, $\left.f^{*}\left(\left.L\right|_{U}\right) \otimes L^{\prime}\right|_{U^{\prime}} ^{-1}=\pi^{*} \beta(\eta)$ holds and since $\beta(\eta)$ is a flat line bundle, $\left.f^{*}\left(\left.L\right|_{U}\right) \otimes L^{\prime}\right|_{U^{\prime}} ^{-1}$ is also a flat line bundle.

Thus all we have to do is showing that the inequality $\operatorname{dim} H^{1}\left(U^{\prime}, \mathcal{O}_{U^{\prime}}\right) \leq \operatorname{dim} H^{1}\left(D^{\prime}, \mathcal{O}_{D^{\prime}}\right)$ holds. Let us consider the short exact sequence $0 \rightarrow I_{D^{\prime}}^{l} / I_{D^{\prime}}^{l+1} \rightarrow \mathcal{O}_{U^{\prime}} / I_{D^{\prime}}^{l+1} \rightarrow \mathcal{O}_{U^{\prime}} / I_{D^{\prime}}^{l} \rightarrow 0$ for $l \geq 1$. Then it follows that the natural map $H^{1}\left(U^{\prime}, \mathcal{O}_{U^{\prime}} / I_{D^{\prime}}^{l+1}\right) \rightarrow H^{1}\left(U^{\prime}, \mathcal{O}_{U^{\prime}} / I_{D^{\prime}}^{l}\right)$ is injective. It is because $H^{1}\left(U^{\prime}, I_{D^{\prime}}^{l} / I_{D^{\prime}}^{l+1}\right)=H^{1}\left(U^{\prime}, I_{D^{\prime}}^{l} \otimes\left(\mathcal{O}_{U^{\prime}} / I_{D^{\prime}}\right)\right)=H^{1}\left(D^{\prime}, \mathcal{O}_{D^{\prime}}\left(-\left.l D^{\prime}\right|_{D^{\prime}}\right)\right)$ vanishes for each $l \geq 1$, since $\mathcal{O}_{D^{\prime}}\left(-K_{D^{\prime}}-\left.l D^{\prime}\right|_{D^{\prime}}\right)=\mathcal{O}_{D^{\prime}}\left(-K_{D^{\prime}}-\left.D^{\prime}\right|_{D^{\prime}}\right) \otimes \mathcal{O}_{D^{\prime}}(-(l-$ 1) $\left.\left.D^{\prime}\right|_{D^{\prime}}\right)$ is nef and big from the assumption. From this combined with the injection in Lemma 3.2, it holds that the natural map $H^{1}\left(U^{\prime}, \mathcal{O}_{U^{\prime}}\right) \rightarrow H^{1}\left(U^{\prime}, \mathcal{O}_{U^{\prime}} / I_{D^{\prime}}\right)=H^{1}\left(D^{\prime}, \mathcal{O}_{D^{\prime}}\right)$ is injective, and thus we obtain the inequality $\operatorname{dim} H^{1}\left(U^{\prime}, \mathcal{O}_{U^{\prime}}\right) \leq \operatorname{dim} H^{1}\left(D^{\prime}, \mathcal{O}_{D^{\prime}}\right)$.

REMARK 3.3. When $\mathcal{O}\left(K_{D}\right)$ is semi-negative, we can prove $H^{1}\left(U^{\prime}, \mathcal{O}_{U^{\prime}}\right) \cong H^{1}\left(D^{\prime}, \mathcal{O}_{D^{\prime}}\right)$ more shortly. Let us consider the short exact sequence $0 \rightarrow I_{D^{\prime}} \rightarrow \mathcal{O}_{U^{\prime}} \rightarrow \mathcal{O}_{U^{\prime}} / I_{D^{\prime}} \rightarrow 0$ and the induced exact sequence $H^{1}\left(U^{\prime}, I_{D^{\prime}}\right) \rightarrow H^{1}\left(U^{\prime}, \mathcal{O}_{U^{\prime}}\right) \rightarrow H^{1}\left(D^{\prime}, \mathcal{O}_{D^{\prime}}\right) \rightarrow H^{2}\left(U^{\prime}, I_{D^{\prime}}\right)$. By the assumption that $\mathcal{O}_{D}\left(-K_{D}\right)=\left.\mathcal{O}_{U}\left(-K_{U}-D\right)\right|_{D}$ is semi-positive and by Ohsawa's theorem [O, 4.5], it follows that the cohomology group $H^{p}\left(U^{\prime}, I_{D^{\prime}}\right)$ vanishes for all $p>0$. Thus $H^{1}\left(U^{\prime}, \mathcal{O}_{U^{\prime}}\right) \cong H^{1}\left(D^{\prime}, \mathcal{O}_{D^{\prime}}\right)$ holds.

Proof of Lemma 3.2. We intrinsically use Rossi's theorem [R, Theorem 3]. Here we remark that, from the assumption that $\mathcal{O}(-D)$ is ample, $U^{\prime}$ is a strongly pseudoconvex domain. Thus, from Rossi's theorem, it turns out that there exists an ideal sheaf $J \subset \mathcal{O}_{U^{\prime}}$ satisfying the condition that (i) $V(J) \subset D^{\prime} \cup\left\{p_{1}, p_{2}, \cdots . p_{l}\right\}$ for some finitely many points $p_{1}, p_{2}, \cdots, p_{l} \in U^{\prime} \backslash D^{\prime}$, where $V(J) \subset U^{\prime}$ stands for the zero set of the ideal sheaf $J$, and that (ii) the natural map $H^{1}\left(U^{\prime}, \mathcal{O}_{U^{\prime}}\right) \rightarrow H^{1}\left(U^{\prime}, \mathcal{O}_{U^{\prime}} / J\right)$ is injective. Here we remark that $H^{1}\left(U^{\prime}, \mathcal{O}_{U^{\prime}} / J\right)=H^{1}\left(D^{\prime}, \mathcal{O}_{U^{\prime}} / J\right)$ holds. It is because the condition (i) and the fact that the first sheaf cohomology vanishes on the zero-dimensional sets $p_{1}, p_{2}, \cdots, p_{l}$.

Let us denote by $I_{D^{\prime}}$ the defining ideal sheaf of $D^{\prime}$, by $I_{p_{j}}$ the defining ideal sheaf of $p_{j}$ for $1 \leq j \leq l$, and by $\hat{J}$ the ideal sheaf $I_{p_{1}} I_{p_{2}} \cdots I_{p_{l}} I_{D^{\prime}}$. By Hilbert's Nullstellensatz, there exists an integer $n$ such that $\hat{J}^{n} \subset J$ holds. Thus the natural map $H^{1}\left(U^{\prime}, \mathcal{O}_{U^{\prime}}\right) \rightarrow H^{1}\left(U^{\prime}, \mathcal{O}_{U^{\prime}} / J\right)$ is decomposed into the composition of two natural maps $H^{1}\left(U^{\prime}, \mathcal{O}_{U^{\prime}}\right) \rightarrow H^{1}\left(U^{\prime}, \mathcal{O}_{U^{\prime}} / \hat{J}^{n}\right)$ and $H^{1}\left(U^{\prime}, \mathcal{O}_{U^{\prime}} / \hat{J}^{n}\right) \rightarrow H^{1}\left(U^{\prime}, \mathcal{O}_{U^{\prime}} / J\right)$. From the condition (ii), it turns out that the map $H^{1}\left(U^{\prime}, \mathcal{O}_{U^{\prime}}\right) \rightarrow H^{1}\left(U^{\prime}, \mathcal{O}_{U^{\prime}} / \hat{J}^{n}\right)$ is also injective, and since $H^{1}\left(U^{\prime}, \mathcal{O}_{U^{\prime}} / \hat{J}^{n}\right)=H^{1}\left(D^{\prime}, \mathcal{O}_{U^{\prime}} / \hat{J}^{n}\right)=H^{1}\left(D^{\prime}, \mathcal{O}_{U^{\prime}} / I_{D^{\prime}}^{n}\right)$ holds, this proves the lemma.

REMARK 3.4. In the above proof of Theorem 1.2, we compared the singular Hermitian metric of $L$ with that of $L^{\prime}$ around the tubular neighborhoods of the divisors. By using this technique, it turns out to be clear that the metric $e^{-\varphi_{L}}$ we constructed above is a minimal singular metric. Moreover, $\varphi_{L^{\prime}}$ in the above proof of Theorem 1.2 can be taken as in Theorem 2.2 when $\left.A\right|_{D}$ is ample, and thus we can conclude that the minimal singular metric we constructed has just the same form as the metric in Theorem 2.2 around $D$ (up to smooth harmonic function). This means that we here determined a minimal singular metric of $L$ around $D$ by only using equilibrium metrics of $\left.t A\right|_{D}+\left.(1-t) L\right|_{D}$ for $0 \leq t \leq 1$ in the above proof in this case.

When $L$ in Theorem 1.2 satisfies that $\left.L\right|_{D}$ is semi-positive, we can say that $L$ is also semi-positive. 
Corollary 3.5. Let $X, D, L$ be those in Theorem 1.2. When $\left.L\right|_{D}$ is semi-positive, $L$ is also semi-positive.

Proof. We use notations in the proof of 1.2, By the proof of Theorem 1.3, it is clear that we can choose smooth $h_{L^{\prime}}$ when $\left.L\right|_{D}$ is semi-positive. We define $\varphi_{L}$ as the function $M\left(\varphi_{A}+\log \left|f_{D}\right|^{2},\left(f^{-1}\right)^{*} \varphi_{L^{\prime}}\right)$ (instead of $\left.\max \left\{\varphi_{A}+\log \left|f_{D}\right|^{2},\left(f^{-1}\right)^{*} \varphi_{L^{\prime}}\right\}\right)$ on each $W_{j} \cap U$, where $M$ is a regularized max function (see [D1, §5.E] for the definition). Then $\left\{e^{-\varphi_{L}}\right\}$ glues up to define a smooth Hermitian metric of $L$ with semi-positive curvature.

We here remark that the idea to use a regularized max function instead of the function "max" is pointed out by Prof. Shin-ichi Matsumura.

\section{SOME EXAMPLES}

4.1. Nef and big line bundles with no locally bounded minimal singular metrics. One can obtain the following corollary immediately from Theorem 1.3 .

Corollary 4.1. Let $X$ be a smooth projective variety, $L$ a nef line bundle over $X$ and let $A$ be an ample line bundle over $X$. Then a minimal singular metric of $L$ is locally bounded if and only if a minimal singular metric of $\mathcal{O}_{\mathbb{P}(A \oplus L)}(1)$ over $\mathbb{P}(A \oplus L)$ is locally bounded.

We remark that the line bundle $\mathcal{O}_{\mathbb{P}(A \oplus L)}(1)$ above is nef and big $([\mathrm{L}, 2.3 .2])$.

ExAmPLE 4.2. Let $(X, L)$ be these in Example 1.7 of [DPS94], which are defined as the relative hyperplane bundle on $X=\mathbb{P}(E)$, where $E$ is a vector bundle defined over an elliptic curve $C$ given by the non-spitting extension $0 \rightarrow \mathcal{O}_{C} \rightarrow E \rightarrow \mathcal{O}_{C} \rightarrow 0$. In this example, $L$ is nef, not big, and possesses no locally-bounded minimal singular metric. Then we can conclude that the nef and big line bundle $\mathcal{O}_{\mathbb{P}(A \oplus L)}(1)$ defined on $\mathbb{P}(L \oplus A)$ for some ample line bundle $A$ on $X$ also has no locally-bounded minimal singular metric. We remark that the similar example is introduced in [BEGZ, 5.4], [F, 5.2].

4.2. Zariski's and Mumford's examples. We can apply Theorem 1.2 to Zariski's and Mumford's examples [L, 2.3.A].

ExAmple 4.3. Let $C \subset \mathbb{P}^{2}$ be a smooth elliptic curve and let $p_{1}, p_{2}, \ldots, p_{12} \in C$ be twelve general points. We define $X$ as the blow up of $\mathbb{P}^{2}$ at these twelve points. We denote by $H$ the pulled back divisor of $X$ of a line in $\mathbb{P}^{2}$ and by $D$ the strict transform of $C$. In this case, since $\left(D^{2}\right)=9-12=-3$ and the genus $g(D)=1$, we can apply Grauert's theorem [G, Satz 7] (see $\S 1$ here) to see that $X, L=\mathcal{O}_{X}(H+D)$, and $D$ satisfy the condition of Theorem [1.2. Moreover, for $\left.L\right|_{D}$ is semi-positive, we can apply Corollary 3.5. Thus $L$ is semi-positive.

There is a generalization of this Zariski's example pointed out by Mumford (see also $[\mathrm{L}, 2.3 .1]$ ). Let $X$ be a smooth projective surface, $A$ a very ample divisor on $X$, and let $D \subset X$ be a curve with $\left(D^{2}\right)<0$ holds and the restriction map $\operatorname{Pic}(X) \rightarrow \operatorname{Pic}(D)$ is injective. We denote by $a, b$ the positive number $(A . D),-\left(D^{2}\right)$, respectively. Then the line bundle $L=\mathcal{O}_{X}(b A+a D)$ is nef, big, satisfying $D \subset \operatorname{Bs}\left|L^{\otimes m}\right|$ for all $m \geq 1$, and there exists a positive integer $p_{0}$ such that $\left|L^{\otimes m} \otimes \mathcal{O}_{X}\left(-p_{0} D\right)\right|$ is generated by global sections for all $m \geq 1$. These $X, L$, and $D$ satisfy the condition of Corollary 3.5 also in this situation when $D$ is smooth, $b$ is sufficiently large, and $p_{0}=1$. Thus, such $L$ is semi-positive, too. 


\section{REFERENCES}

[BeGZ] S. Boucksom, P. Eyssidieux, V. Guedj, A. Zeriahi, Monge-Ampère equations in big cohomology classes. Acta Math. 205 (2010), 199-262.

[D1] J.-P. Demailly, Complex Analytic and Differential Geometry, monograph, 2012, available at http://www-fourier.ujf-grenoble.fr/ demailly.

[D2] J.-P. Demailly, Analytic methods in algebraic geometry, Surveys of Modern Mathematics, 1. International Press, Somerville, MA, 2012.

[DPS94] J-P. Demailly, T. Peternell, M. Schneider, Compact complex manifolds with numerically effective tangent bundles, J. Algebraic Geom., 3 (1994), 295-345

[DPS00] J.-P. Demailly, T. Peternell, M. Schneider, Pseudo-effective line bundles on compact Kähler manifolds, Internat. J. Math. 12 (2001), 689-741.

[F] O. Fujino, A transcendental approach to Kollár's injectivity theorem II, arXiv:math/0705.1199v4.

[G] H. Grauert, Über Modifikationen und exzeptionelle analytische Mengen, Math. Ann. 146 (1962), 331-368.

[L] R. Lazarsfeld, Positivity in algebraic geometry. I, Ergebnisse der Mathematik und ihrer Grenzgebiete. 3. Folge. A Series of Modern Surveys in Mathematics [Results in Mathematics and Related Areas. 3rd Series. A Series of Modern Surveys in Mathematics], 48. Springer-Verlag, Berlin, 2004.

[O] T. OhsawA, Vanishing theorems on complete Kähler manifolds, Publ. Res. Inst. Math. Sci. 20 (1984), no. 1, 2138.

[R] H. Rossi, Strongly pseudoconvex manifolds, Lectures in Modern Analysis and Applications I, Lecture Notes in Mathematics 103, (1969), 10-29.

Graduate School of Mathematical Sciences, The University of Tokyo

3-8-1 Komaba, Meguro-ku, Tokyo, 153-8914

JAPAN

E-mail address: tkoike@ms.u-tokyo.ac.jp 